\title{
Evaluating the efficacies of Maximum Tolerated Dose and metronomic chemotherapies: A mathematical approach
}

\author{
Rafael T. Guiraldello ${ }^{\mathrm{a}, *}$, Marcelo L. Martins ${ }^{\mathrm{b}}$, Paulo F.A. Mancera ${ }^{\mathrm{a}, *}$ \\ a Departamento de Bioestatística, Universidade Estadual Paulista "Júlio de Mesquita Filho", Botucatu 18618-689, Brazil \\ ${ }^{\mathrm{b}}$ Departamento de Física, Universidade Federal de Viçosa, Viçosa 36570-900, Brazil
}

\section{H I G H L I G H T S}

- A 1D model based on two chemotherapeutic protocols, Maximum Tolerated Dose (MTD) and metronomic.

- Two methods of drug delivery are considered and analyzed.

- The metronomic protocol proves more effective in prolonging the patient's life than MTD.

\section{A R T I C L E I N F O}

\section{Article history:}

Received 22 December 2015

Received in revised form 11 March 2016

Available online 30 March 2016

\section{Keywords:}

Mathematical oncology

Cancer

Tumor-induced angiogenesis

Chemotherapy

\begin{abstract}
A B S T R A C T
We present a mathematical model based on partial differential equations that is applied to understand tumor development and its response to chemotherapy. Our primary aim is to evaluate comparatively the efficacies of two chemotherapeutic protocols, Maximum Tolerated Dose (MTD) and metronomic, as well as two methods of drug delivery. Concerning therapeutic outcomes, the metronomic protocol proves more effective in prolonging the patient's life than MTD. Moreover, a uniform drug delivery method combined with the metronomic protocol is the most efficient strategy to reduce tumor density.
\end{abstract}

(C) 2016 Elsevier B.V. All rights reserved.

\section{Introduction}

Today, cancer has replaced heart disease as the leading cause of death among American citizens under the age of 85 [1] and will likely become the leading killer in some other parts of the world within a few years [2]. Nevertheless, cancer can be prevented by avoiding certain risk factors and can be cured via chemotherapy, radiotherapy, surgery or immunotherapy, for example, if detected in its early stages [3].

Often, cancer treatment involves chemotherapy, which aims to eliminate the tumor or control the growth of its cells via the administration of one or more drugs. Frequently, chemotherapy is based on the Maximum Tolerated Dose (MTD) protocol. MTD is designed to administer the maximum amount of anti-cancer drugs tolerated by a patient. In consequence, MTD treatments produce severe side effects, such as diarrhea, vomiting and hair loss. Hence, a period of rest, normally lasting 3 to 4 weeks, is necessary to allow the patient to recover. However, this rest period also allows cancer cells to regrow and develop drug resistance, which can reduce the efficacy of MTD over time. As an alternative to MTD, metronomic chemotherapy is currently in development. Metronomic chemotherapy consists of administering comparatively low doses of drugs on a frequent or continuous schedule and without extended periods of rest [4]. Clinically, a major challenge posed

\footnotetext{
* Corresponding authors.

E-mail addresses: rafaeltrevisanuto@gmail.com (R.T. Guiraldello), mmartins@ufv.br (M.L. Martins), pmancera@ibb.unesp.br (P.F.A. Mancera).
} 
by metronomic chemotherapy is to define the optimum drug doses and the intervals of drug infusion in a manner that is rational rather than empirical $[5,6]$.

Regardless of the chemotherapeutic approach adhered to, fundamental issues and technical hurdles must be understood and overcome in order to enhance efficacy. It is imperative to enlarge our current understanding of the complex and interrelated dynamics of the growing tumor, its microenvironment and chemotherapeutic agents. The nonlinearities and complexities inherent to such dynamics indicate the need for mathematical approaches. Quantitative models can reveal the major parameters affecting therapeutic outcomes, guide new assays by indicating relevant physiological processes for further investigation and reduce the amount of experimentation necessary to develop effective treatments. Many mathematical frameworks have been considered in order to model tumor growth; for example, ordinary and partial differential equations [7,8], cellular automata [9-11], optimization [12-14] and multi-scale modeling [15,16]. Concerning cancer treatment, several mathematical models have provided valuable insights about the efficacy of combined therapies based on antiangiogenic agents and cytotoxic drugs [17-19]. Differential equation models (ordinary (ODE) and partial (PDE)) were presented by Ref. [20], in which comparisons between different combinations of drugs and dosage schedules were performed. Andre et al. [5] proposed a PDE model to analyze the interaction dynamics of tumor, normal, necrotic and endothelial cells, as well as the influence of oxygen, angiogenic factors and chemotherapy protocols (MTD and metronomic) on these cells. The MTD and metronomic protocols were implemented in discrete models studied by Ref. [21]. Through transport equation models [22], the actions of MTD and metronomic chemotherapy on metastatic and primary tumors were investigated. The drug Temozolomide was considered in a model used by Ref. [23] to compare MTD and metronomic protocols. Rodrigues et al. [24] compared a standard protocol of chemotherapy with metronomic chemotherapy, focusing on the antiangiogenic efficiency of metronomic chemotherapy in preventing tumor neovascularization.

In the present paper, we use a mathematical model to study antiangiogenic chemotherapeutic treatments and understand how they work. Specifically, we create a mathematical model of tumor growth in which (i) normal and cancer cells compete for resources; (ii) the malignant cells induce the proliferation of endothelial cells in order to enlarge the tissue carrying capacity for the growing solid tumor; and (iii) the chemotherapeutic drug, administered according to either the metronomic [5,14,20,24-26] or the usual MTD schedules, has, in addition to cytotoxic, antiangiogenic effects. The use of this model was motivated by Ref. [24], where the authors propose a system of ODEs to analyze the administration of a cycle-nonspecific chemotherapeutic drug on a tumor, which also acts on vascular endothelial cells, impairing angiogenesis and decreasing the carrying capacity for tumor cells. The present paper is structured as follows: in Section 2, we present the model and chemotherapy protocols; some theoretical results and numerical simulations are presented in Section 3 and discussed in Section 4; finally, we make some concluding remarks in Section 4.

\section{Model}

We propose a continuous mathematical model formulated in terms of PDEs to describe the growth of a solid tumor. The model takes into account the competition between normal and tumor cells, the growth of endothelial cells induced by the malignant cells to increase the tumoral carrying capacity (angiogenesis) and the action of a chemotherapeutic agent on all cell populations. Our approach is based on a model proposed in Ref. [27] (see also Ref. [24]). We assume that normal cells, cancer cells, endothelial cells, and the chemotherapeutic agent move randomly (normal diffusion). Moreover, the endothelial cells migrate by chemotaxis in response to tumor angiogenic factors (TAFs) released by the tumor. Denoting the densities of tumor, normal, and endothelial cells by $N_{1}, N_{2}$ and $L_{1}$, respectively, and the concentration of the chemotherapeutic agent by $Q$, the model equations are

$$
\left\{\begin{array}{l}
\frac{\partial N_{1}}{\partial t}=D_{1} \nabla^{2} N_{1}+r_{1} N_{1}\left(1-\frac{N_{1}+\alpha_{12} N_{2}}{k_{1}+L_{1}}\right)-N_{1} \mu \frac{Q}{a+Q} \\
\frac{\partial N_{2}}{\partial t}=D_{2} \nabla^{2} N_{2}+r_{2} N_{2}\left(1-\frac{N_{2}+\alpha_{21} N_{1}}{k_{2}}\right)-N_{2} v \frac{Q}{b+Q} \\
\frac{\partial L_{1}}{\partial t}=D_{L} \nabla^{2} L_{1}+\xi N_{1}\left(1-\frac{L_{1}}{k_{L}}\right)-\frac{\sigma}{k_{2}} L_{1}^{2}-L_{1} \eta \frac{Q}{c+Q}-\nabla \cdot\left(\bar{\chi}_{\left(N_{1}, L_{1}\right)} \nabla N_{1}\right) \\
\frac{\partial Q}{\partial t}=D_{3} \nabla^{2} Q+q-\lambda Q,
\end{array}\right.
$$

where $r_{i}>0(i=1,2)$ denote the growth rates of the tumor and normal populations, $k_{i}$ are their corresponding carrying capacities, $D_{i}$ are their diffusivities; $k_{1}+L_{1}$ is the carrying capacity of the tumor cells, which varies with the endothelial cell density due to the nutritional capacity granted to the tumor by the new vascularization, and $\alpha_{i j}$ is the competition coefficient of cell type $j$ on type $i$. Indeed, the growth of endothelial cells is induced by tumor cells at an intrinsic rate $\xi$, which models angiogenesis (sprouting from pre-existing vessels) and vascular mimicry (tumor cells mimicking endothelial cell functions) $[28,29]$. Cancer stem cells (CSC) can differentiate into endothelial cells and these tumor derived endothelial cells form tumor blood vessels [30]. Also, CSCs are the major producers of vascular endothelial growth factor (VEGF) and stromal-derived factor 1 (SDF-1) in the tumor, leading to a strong angiogenic response [31]. The carrying capacity $k_{L}$ is related to tissue perfusion and the endothelial cells compete intra specifically for nutrients at a rate $\sigma$, have a diffusivity $D_{L}$ and exhibit chemotactic migration. The parameter representing this chemotaxis is $\bar{\chi}_{\left(N_{1}, L_{1}\right)}=\chi L_{1} /\left(k_{2}+N_{1}\right)$, which 
assumes decreasing chemotactic sensitivity with increasing TAF concentration [32]. The TAF concentration is proportional to the neoplastic cell density.

Finally, the infusion rate of the chemotherapeutic agent is modeled by the function $q$ defined in [ $0, \infty)$ and satisfying $q(\cdot) \geq 0$. As in Ref. [33], we define the rate of drug infusion as

$$
q= \begin{cases}z, & n<t \leq n+\tau \\ 0, & n+\tau<t \leq n+T\end{cases}
$$

where $T$ is the cycle time interval, $n=0, T, 2 T, \ldots$ and $\tau$ is the infusion time with $T \gg \tau$ (administration in bolus doses). $\lambda>0$ is the clearance rate of the chemotherapeutic drug and $D_{3}$ its diffusivity. The drug's effect on cell populations is modeled by a Michaelis-Menten-type functional response, where $a, b$ and $c$ determine the saturation of the drug; $\mu$ and $v$ are, respectively, the mortality rates of tumor and normal cells due to the treatment; and $\eta$ models the intensity of the antiangiogenic effect due to the chemotherapeutic agent.

\subsubsection{Uniform drug administration}

In this model of drug administration, it is assumed that the total amount of the drug, $\bar{q}$, is distributed uniformly over the entire volume $\Omega$ of affected tissue. Therefore, the rate of drug infusion is

$$
z(x, t)=\frac{\bar{q}}{\Omega \tau},
$$

where $x \in \Omega$.

\subsubsection{Density-dependent drug administration}

In this mode of drug administration, it is assumed that the rate of drug infusion is

$$
z(x, t)=\frac{\bar{q}}{\tau}\left(\int_{\Omega} L_{1}(x, t) d x\right)^{-1} L_{1}(x, t),
$$

where $x \in \Omega$. Therefore, the total amount of the drug administered per infusion, $\bar{q}$, is distributed through the area in proportion to the local density of the endothelial cells. Accordingly, more of the drug is delivered where the vascularization is denser.

\subsubsection{Non-dimensional model}

Considering $\Omega=(0, L)$ and introducing the non-dimensional variables

$$
\tilde{N}_{1}=\frac{N_{1}}{k_{2}}, \quad \tilde{N}_{2}=\frac{N_{2}}{k_{2}}, \quad \tilde{Q}=\frac{Q}{k_{2}}, \quad \tilde{L_{1}}=\frac{L_{1}}{k_{2}}, \quad \tilde{x}=\frac{x D_{2}}{L^{3} r_{1}}, \quad \tilde{t}=r_{1} t,
$$

and the non-dimensional parameters

$$
\begin{aligned}
& \tilde{D}_{1}=\frac{D_{1} D_{2}^{2}}{L^{6} r_{1}^{3}}, \quad \tilde{\mu}=\frac{\mu}{r_{1}}, \quad \tilde{D}_{2}=\frac{D_{2}^{3}}{L^{6} r_{1}^{3}}, \quad \tilde{r}_{2}=\frac{r_{2}}{r_{1}}, \quad \tilde{v}=\frac{v}{r_{1}}, \quad \tilde{D}_{3}=\frac{D_{3} D_{2}^{2}}{L^{6} r_{1}^{3}}, \\
& \tilde{d}=\frac{1}{k_{2} r_{1}}, \quad \tilde{\lambda}=\frac{\lambda}{r_{1}}, \quad \tilde{D}_{L}=\frac{D_{L} D_{2}^{2}}{L^{6} r_{1}^{3}}, \quad \tilde{\xi}=\frac{\xi}{r_{1}}, \quad \tilde{\sigma}=\frac{\sigma}{r_{1}}, \quad \tilde{\eta}=\frac{\eta}{r_{1}}, \\
& \tilde{\chi}=\frac{\chi D_{2}^{2}}{L^{6} r_{1}^{3}}, \quad \tilde{k}_{1}=\frac{k_{1}}{k_{2}}, \quad \tilde{k}_{L}=\frac{k_{L}}{k_{2}}, \quad \tilde{a}=\frac{a}{k_{2}}, \quad \tilde{b}=\frac{b}{k_{2}}, \quad \tilde{c}=\frac{c}{k_{2}} .
\end{aligned}
$$

Eqs. (1) can be written as the non-dimensional system

$$
\left\{\begin{array}{l}
\frac{\partial N_{1}}{\partial t}=D_{1} \nabla^{2} N_{1}+N_{1}\left(1-\frac{N_{1}+\alpha_{12} N_{2}}{k_{1}+L_{1}}\right)-N_{1} \mu \frac{Q}{a+Q} \\
\frac{\partial N_{2}}{\partial t}=D_{2} \nabla^{2} N_{2}+r_{2} N_{2}\left(1-N_{2}-\alpha_{21} N_{1}\right)-N_{2} v \frac{Q}{b+Q} \\
\frac{\partial L_{1}}{\partial t}=D_{L} \nabla^{2} L_{1}+\xi N_{1}\left(1-\frac{L_{1}}{k_{L}}\right)-\sigma L_{1}^{2}-L_{1} \eta \frac{Q}{c+Q}-\nabla \cdot\left(\frac{\chi L_{1}}{1+N_{1}} \nabla N_{1}\right) \\
\frac{\partial Q}{\partial t}=D_{3} \nabla^{2} Q+d q-\lambda Q,
\end{array}\right.
$$

where the tildes were omitted for simplicity. 


\subsubsection{Boundary and initial conditions}

Let $\Omega=(0, L)$, with $L=1$, and the boundary conditions on $\partial \Omega$ given by

$$
\frac{\partial N_{1}}{\partial n}=\frac{\partial N_{2}}{\partial n}=\frac{\partial Q}{\partial n}=\frac{\partial L_{1}}{\partial n}=0,
$$

where $\frac{\partial}{\partial n} \equiv \hat{n} \cdot \nabla$ is the normal derivative along $\hat{n}$, the outward unit normal vector to $\Omega$.

We consider an avascular tumor in the center of the domain, and then the initial conditions are

$$
\left\{\begin{array}{l}
N_{1}(0, x)=0.1 k_{1} \mathrm{e}^{-20\|x-0.5\|^{2}} \\
N_{2}(0, x)=1-N_{1} \\
L_{1}(0, x)=0 \\
Q(0, x)=0 .
\end{array}\right.
$$

It should be reminded that $L_{1}$ refers to the abnormal neovasculature induced by the tumor growth. Accordingly, the initial value $L_{1}(0, x)=0$ is assumed.

\section{Results}

\subsection{Linear stability analysis}

In this subsection, we analyze the model equations (6) when spatial fluctuations and correlations are neglected. In this case, the system (6) is reduced to the following ODEs:

$$
\left\{\begin{array}{l}
\frac{\mathrm{d} N_{1}}{\mathrm{~d} t}=N_{1}\left(1-\frac{N_{1}+\alpha_{12} N_{2}}{k_{1}+L_{1}}\right)-N_{1} \mu \frac{Q}{a+Q} \\
\frac{\mathrm{d} N_{2}}{\mathrm{~d} t}=r_{2} N_{2}\left(1-N_{2}-\alpha_{21} N_{1}\right)-N_{2} v \frac{Q}{b+Q} \\
\frac{\mathrm{d} L_{1}}{\mathrm{~d} t}=\xi N_{1}\left(1-\frac{L_{1}}{k_{L}}\right)-\sigma L_{1}^{2}-L_{1} \eta \frac{Q}{c+Q} \\
\frac{d Q}{\mathrm{~d} t}=d q-\lambda Q .
\end{array}\right.
$$

\subsubsection{Model without treatment}

In the absence of a chemotherapeutic treatment, $Q=q=0$ and the system (9) becomes

$$
\left\{\begin{array}{l}
\frac{\mathrm{d} N_{1}}{\mathrm{~d} t}=N_{1}\left(1-\frac{N_{1}}{k_{1}+L_{1}}\right)-\alpha_{12} \frac{N_{1} N_{2}}{k_{1}+L_{1}} \\
\frac{\mathrm{d} N_{2}}{\mathrm{~d} t}=r_{2} N_{2}\left(1-N_{2}\right)-\alpha_{21} r_{2} N_{2} N_{1} \\
\frac{\mathrm{d} L_{1}}{\mathrm{~d} t}=\xi N_{1}\left(1-\frac{L_{1}}{k_{L}}\right)-\sigma L_{1}^{2} .
\end{array}\right.
$$

This system has the following equilibrium points:

$$
\begin{aligned}
& P_{1}\left(N_{1}, N_{2}, L_{1}\right)=(0,0,0), \quad P_{2}\left(N_{1}, N_{2}, L_{1}\right)=(0,1,0), \\
& P_{3}\left(N_{1}, N_{2}, L_{1}\right)=\left(N_{1}^{*}, 0, N_{1}^{*}-k_{1}\right), \\
& P_{4}\left(N_{1}, N_{2}, L_{1}\right)=\left(N_{1}^{*}, 1-\alpha_{21} N_{1}^{*}, N_{1}^{*}-k_{1}+\alpha_{12}\left(1-\alpha_{21} N_{1}^{*}\right)\right),
\end{aligned}
$$

with

$$
N_{1}^{*}=\frac{1}{2} \frac{\xi\left(k_{1}+k_{L}\right)+2 \sigma k_{1} k_{L}+\sqrt{\xi^{2}\left(k_{1}+k_{L}\right)^{2}+4 \xi \sigma k_{1} k_{L}^{2}}}{\left(\sigma k_{L}+\xi\right)} .
$$

The equilibrium solution $N_{1}^{*}$, given by $N_{1}^{*}=k_{1}+L_{1}^{*}$, implies in a second degree polynomial equation for $L_{1}^{*}$. The solution for $L_{1}^{*}$ involving a negative square root is always negative, which is biologically unacceptable since $L_{1}^{*}$ is a population density. In consequence, there is only one $N_{1}^{*}$ corresponding to the $L_{1}^{*}$ containing the positive square root.

The point $P_{1}$ is the trivial and represents the extinction of all cell populations; $P_{2}$ corresponds to the extinction of the malignant and endothelial cells and can be biologically interpreted as a spontaneous cure; and $P_{3}$ is associated with the extinction of normal cells in the presence of tumor and endothelial cells. Biologically, the normal tissue was absolutely disrupted by the neoplastic mass and its abnormally induced neovasculature. Finally, $P_{4}$ represents the coexistence of all cell populations and, therefore, less aggressive tumor growth. 

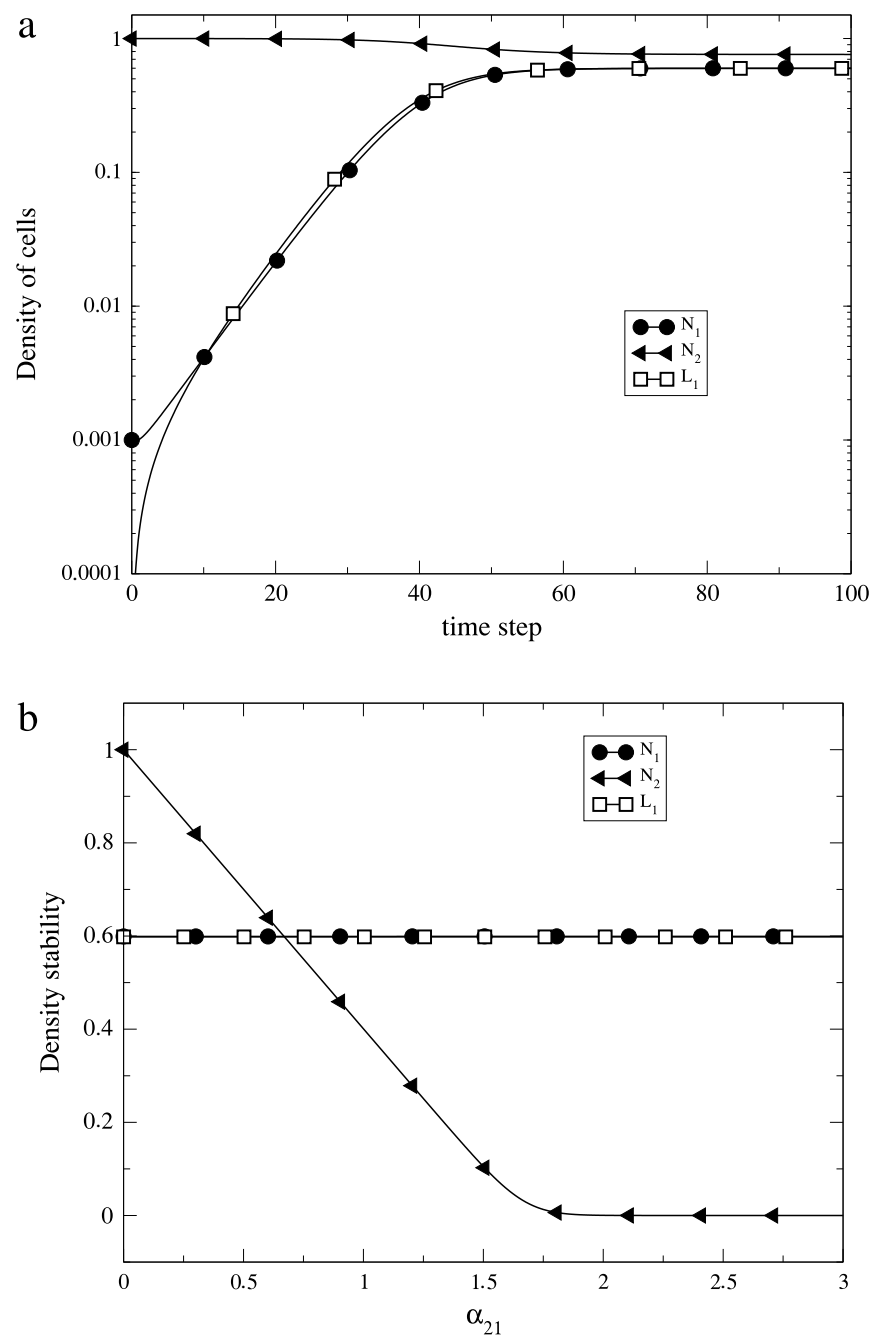

Fig. 1. (a) Progress curves converging to the fixed coexistence point $P_{4}$. Parameters: $\alpha_{12}=10^{-4}, \alpha_{21}=4 \times 10^{-1}, r_{2}=2 \times 10^{-1}, \xi=2 \times 10^{-1}, k_{1}=10^{-3}$, $k_{L}=6 \times 10^{-1}$ and $\sigma=10^{-3}$. (b) Bifurcation diagram for the parameter $\alpha_{21}$. The numerical integrations were performed using the fourth-order Runge-Kutta method.

In $P_{1}$, the Jacobian matrix of (10) has the eigenvalues $\tau_{1}=0, \tau_{2}=1$ and $\tau_{3}=r_{2}>0$. Consequently, $P_{1}$ is an unstable fixed point. At $P_{2}$, these eigenvalues are $\tau_{1}=0, \tau_{2}=-r_{2}$, and $\tau_{3}=\left(k_{1}-\alpha_{12}\right) / k_{1}$. Therefore, if $k_{1}>\alpha_{12}, P_{2}$ is unstable. Otherwise, it is marginally stable. In turn, at $P_{3}$ the eigenvalues are $\tau_{1}=r_{2}\left(1-\alpha_{21} N_{1}^{*}\right), \tau_{2}$ and $\tau_{3}$, with $\operatorname{Re}\left(\tau_{2}\right)$ and $\operatorname{Re}\left(\tau_{3}\right)<0$. Hence, if $\alpha_{21}>\frac{1}{N_{1}^{*}}, P_{3}$ is stable. Otherwise, it is unstable. Finally, regarding the coexistence point $P_{4}$, we were unable to obtain an analytical result regarding its stability, which was inferred through numerical simulations.

Fig. 1(a) illustrates a typical cancer progression converging towards the coexistence fixed point $P_{4}$. In Fig. 1(b), the bifurcation diagram for the parameter $\alpha_{21}$ is shown. These simulations and the analytical results for $P_{3}$ indicate that there is a critical value, $\alpha_{c}$, which divides the parameter space into two parts: one attracted by $P_{3}$ and the other by $P_{4}$. Thus, bistability between the points $P_{3}$ and $P_{4}$ is not possible.

Interestingly, if normal cells are strong competitors, implying $\alpha_{12}>k_{1}$, tumor eradication without treatment is possible only if the density of the cancer cells is very low and neovascularization is insufficient to significantly increase the tumors carrying capacity. From an ecological viewpoint, this scenario can be associated with micrometastases that fail to colonize "hostile' sites or avascular primary tumors that recede completely, owing to their incapacity to promote adequate angiogenesis.

\subsubsection{Model with treatment}

At this point, the spatially homogeneous system (9) must be analyzed. Its equilibrium points are:

$$
\begin{aligned}
& P_{1}\left(N_{1}, N_{2}, L_{1}, Q\right)=\left(0,0,0, Q^{*}\right), \quad P_{2}\left(N_{1}, N_{2}, L_{1}, Q\right)=\left(0, N_{2}^{*}, 0, Q^{*}\right), \\
& P_{3}\left(N_{1}, N_{2}, L_{1}, Q\right)=\left(N_{1}^{*}, 0, L_{1}^{*}, Q^{*}\right), \quad P_{4}\left(N_{1}, N_{2}, L_{1}, Q\right)=\left(\hat{N}_{1}, \hat{N}_{2}, \hat{L}_{1}, Q^{*}\right),
\end{aligned}
$$


Table 1

Parameters for the model without treatment.

\begin{tabular}{llll}
\hline Parameters & Values & Unity & Reference \\
\hline$D_{1}$ & $10^{-4}$ & $(\mathrm{~mm})^{2} \times$ day $^{-1}$ & {$[34]$} \\
$D_{2}$ & $10^{-5}$ & $(\mathrm{~mm})^{2} \times$ day $^{-1}$ & $D_{1}>D_{2}$ \\
$D_{L}$ & $10^{-5}$ & $(\mathrm{~mm})^{2} \times$ day $^{-1}$ & $D_{L} \sim D_{2}$ \\
$r_{1}$ & $10^{-2}$ & day $^{-1}$ & {$[35]$} \\
$r_{2}$ & $10^{-3}$ & day $^{-1}$ & $r_{2}<r_{1}$ \\
$\xi$ & $2 \times 10^{-3}$ & day $^{-1}$ & {$[36]$} \\
$\sigma$ & $10^{-5}$ & day $^{-1}$ & {$[36]$} \\
$\alpha_{12}$ & $9 \times 10^{-5}$ & - & {$[24]$} \\
$\alpha_{21}$ & $9 \times 10^{-2}$ & - & {$[24]$} \\
$k_{1}{ }^{a}$ & $10^{8}$ & mass $\times \mathrm{mm}^{-1}$ & {$[37]$} \\
$k_{2}$ & $10^{12}$ & mass $\times \mathrm{mm}^{-1}$ & $k_{2} \sim k_{1}$ \\
$k_{L}$ & $8 \times 10^{11}$ & mass $\times \mathrm{mm}^{-1}$ & - \\
$\chi$ & $3.8 \times 10^{-3}$ & $(\mathrm{~mm})^{2} \times$ day $^{-1}$ & {$[32]$} \\
\hline${ }^{a}$ Unity conversion is based on Refs. [38,37]. & &
\end{tabular}

where

$$
\begin{aligned}
N_{2}^{*} & =1-v d \frac{q}{r_{2}(b \lambda+d q)}, \quad \mu^{*}=1-\mu d \frac{q}{a \lambda+d q}, \quad \eta^{*}=\eta d \frac{q}{c \lambda+d q}, \\
L_{1}^{*} & =\frac{1}{2} \frac{\xi \mu^{*}\left(k_{L}-k_{1}\right)-\eta^{*} k_{L}+\sqrt{\Delta}}{\left(\xi \mu^{*}+\sigma k_{L}\right)}, \\
\Delta & =\left[\xi \mu^{*}\left(k_{L}-k_{1}\right)-\eta^{*} k_{L}\right]^{2}+4\left(\xi \mu^{*}+\sigma k_{L}\right)\left(\xi \mu^{*} k_{1} k_{L}\right), \\
Q^{*} & =d \frac{q}{\lambda}, \\
N_{1}^{*} & =\left(L_{1}^{*}+k_{1}\right) \mu^{*} .
\end{aligned}
$$

$P_{1}$ describes the extinction of all cell populations caused by the chemotherapeutic agent. At $P_{2}$, tumor and endothelial cells are both eliminated, and this fixed point is interpreted as the cure due to chemotherapy. The fixed point $P_{3}$, at which the density of normal cells goes to zero, represents a completely unsuccessful chemotherapeutic treatment. Finally, at $P_{4}$, the coexistence among all cell populations occurs under treatment. This scenario corresponds to a therapy that only halts the growth of a solid tumor, eventually eliciting its partial remission.

Concerning the stability of such fixed points, the eigenvalues of the Jacobian matrix evaluated in $P_{1}$ are $\tau_{1}=-\lambda, \tau_{2}=$ $-\eta^{*}, \tau_{3}=r_{2} N_{2}^{*}$ and $\tau_{4}=\mu^{*}$. Since $\tau_{3}>0$ and $\tau_{4}>0, P_{1}$ is unstable. At $P_{2}$, the eigenvalues are $\tau_{1}=-\lambda, \tau_{2}=$ $-\eta^{*}, \tau_{3}=-r_{2} N_{2}^{*}$ and $\tau_{4}=\mu^{*}-\frac{\alpha_{12} N_{2}^{*}}{k_{1}}$. Since the first three eigenvalues are real and negative, the stability of $P_{2}$ depends exclusively on $\tau_{4}$. Therefore, if $\alpha_{12}<\frac{\mu^{*} k_{1}}{N_{2}^{*}}, P_{2}$ is unstable. Otherwise, it is a stable, fixed point. At $P_{3}$, the eigenvalues are $\tau_{1}=-\lambda, \tau_{2}=r_{2}\left(N_{2}^{*}-\alpha_{21} N_{1}^{*}\right), \tau_{3}$ and $\tau_{4}$, with $\operatorname{Re}\left(\tau_{3}\right)$ and $\operatorname{Re}\left(\tau_{4}\right)$ negative. Thus, the stability of $P_{3}$ depends on $\tau_{2}$. As a result, if $\alpha_{21}>\frac{N_{2}^{*}}{N_{1}^{*}}, P_{3}$ is stable. Otherwise, it is an unstable, fixed point. Therefore, if the malignant cells are strong competitors, the normal cells are eliminated, despite chemotherapy. Conversely, if the normal cells are strong competitors, the chemotherapeutic treatment can eradicate the tumor very quickly.

Again, we were unable to obtain an analytical result for the stability of $P_{4}$, which was therefore inferred through numerical simulations.

Fig. 2(a) shows an example of cancer progression leading to the coexistence point $P_{4}$. In Fig. 2(b), the bifurcation diagram at parameter $\alpha_{21}$ exhibits the transition from $P_{4}$ to $P_{3}$. Again, numerical simulations and the analytical results for $P_{3}$ indicate that there is a critical value, $\alpha_{c}$, that divides the parameter space into two parts, both having only one attractor, thus excluding the possibility of bistability.

\subsection{Numerical simulations}

In this subsection, we analyze the spatially explicit model equations (6) through numerical simulations on $\Omega=(0,1)$.

\subsubsection{Cancer progression without treatment}

In Fig. 3(a), a log-linear plot of normal, cancer and endothelial cell densities as a function of time illustrates tumor growth without treatment. The parameters used are listed in Table 1. Observe that during a short initial period, the tumor cells induce fast endothelial growth (angiogenesis). After this initial period, the densities of cancer and endothelial cells experience a phase of exponential growth prior to saturation. Furthermore, as emphasized in Fig. 3(b), the phase of exponential growth begins when the endothelial cell density surpasses the density of malignant cells, demonstrating the relevance of neovascularization in the promotion of tumor growth. 

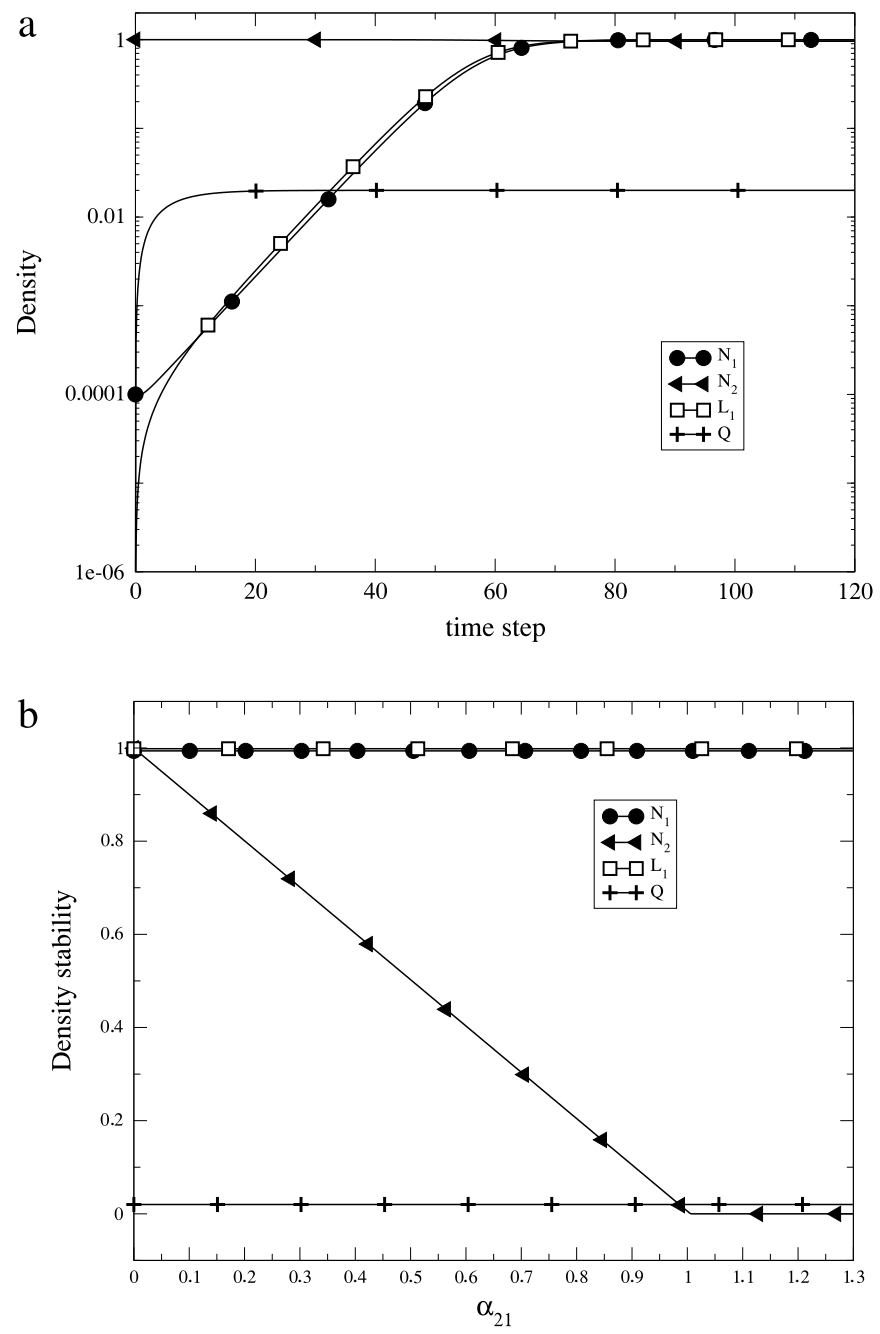

Fig. 2. (a) An example of cancer progression reaching the coexistence point $P_{4}$. Parameters: $\alpha_{12}=10^{-5}, \alpha_{21}=4 \times 10^{-2}, r_{2}=2 \times 10^{-1}, \xi=2 \times 10^{-1}, k_{1}=$ $10^{-4}, k_{L}=1, d=10^{-2}, \lambda=2 \times 10^{-1}, q=8 \times 10^{-1}, a=5 \times 10^{-1}, b=10^{-5}, c=10^{-5}, \mu=6 \times 10^{-2}, v=10^{-4}, \eta=2 \times 10^{-1}$ and $\sigma=10^{-4}$. (b) Bifurcation diagram for the parameter $\alpha_{21}$.

\subsubsection{Effects of chemotherapy}

In this section, the effects of chemotherapy on tumor growth are discussed. The focus of our analysis lies in the comparison between the therapeutic efficacies of MTD and metronomic protocols.

We simulated two programs of chemotherapy. The first was an MTD protocol consisting of four drug administrations, each 21 days in duration [24]. For this treatment, the parameters used in Eq. (2) were $\bar{q}=7200$ (see Ref. [27]) for the drug load, $T=21$ for the administration interval, and $n=0,1,2$ and 3. Secondly, a metronomic protocol, for which $\bar{q}=3600$ (half of the MTD value), $T=6$ and $n=0,1,2, \ldots 15$, corresponding to 16 infusions performed in 6-day intervals [24]. The values adopted for the other parameters in the simulations are listed in Table 2. Furthermore, for both chemotherapeutic protocols, we also tested the two methods of the drug delivery: uniform and density-dependent.

The primary results obtained from these simulations are shown in Fig. 4. During treatment, the metronomic protocol is more efficient in reducing tumor cell density than the MTD protocol (see Fig. 4(a)). Regarding drug delivery, whatever the chemotherapeutic protocol, a uniform method is the most efficient. Consequently, metronomic chemotherapy with uniform drug delivery is the strategy of greatest efficacy in reducing the tumor cell density. However, although less efficient in reducing the tumor density, density-dependent drug delivery most strongly delays cancer relapse after failed chemotherapy, be it MTD or metronomic. This is shown in Fig. 4(b).

In Figs. 5 and 6, the spatial density distributions of tumor and endothelial cells during treatment are exhibited at distinct times for the MTD and metronomic protocols, respectively. As expected, for both MTD and metronomic chemotherapies in a density-dependent drug delivery mode, the local densities of cancer cells decline faster where the densities of endothelial cells are higher. In contrast, for a uniform drug delivery, the local densities of cancer cells decay at the same rate everywhere. Counter-intuitively, where the initial density of endothelial cells is low (in our simulations, near the tissue borders), these 

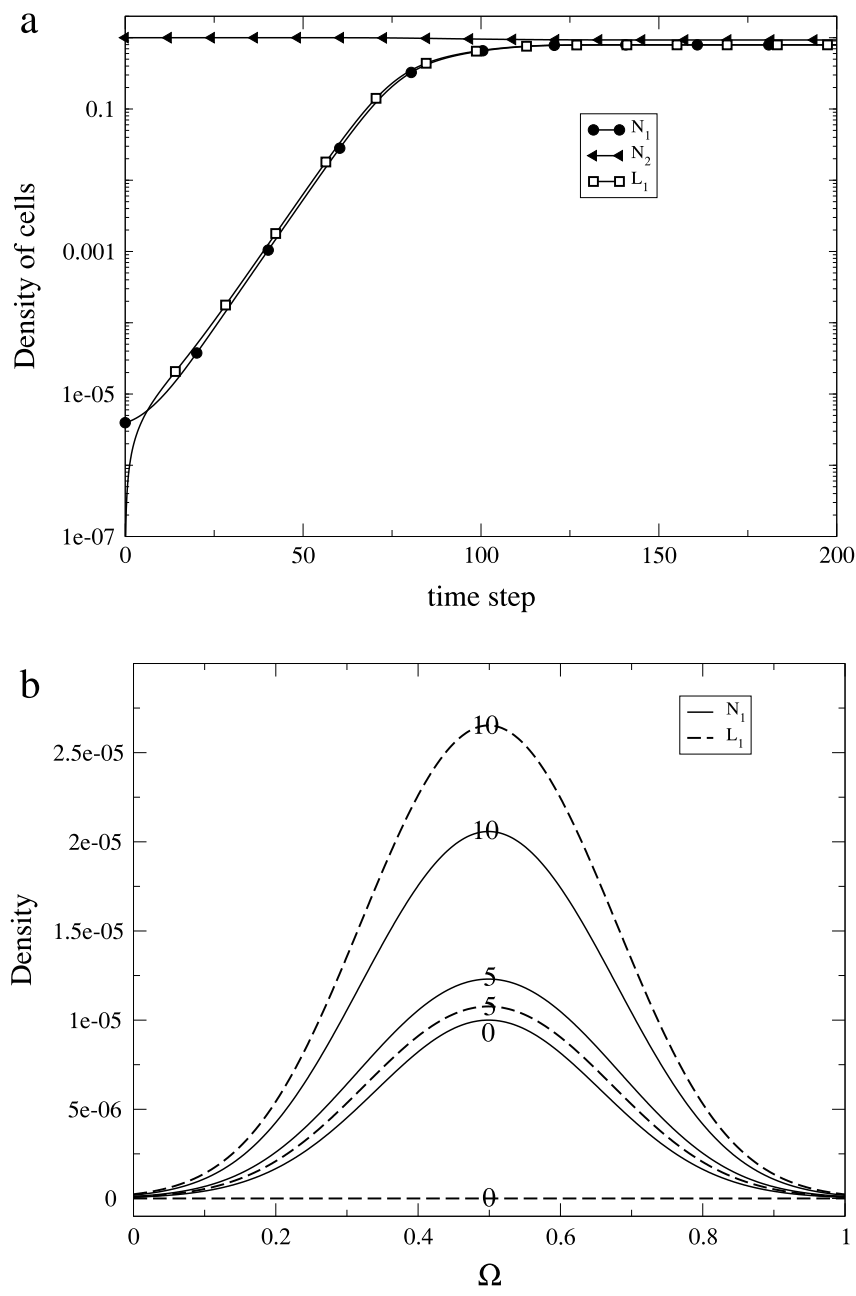

Fig. 3. (a) Evolution in time of the cell densities on the interval $\bar{\Omega}=[0,1]$. (b) Spatial distribution of cancer and endothelial cell densities at three different times (indicated above the curves). At the beginning $(t=0)$, the density of cancer cells is assumed to follow a Gaussian distribution centered at $x=0.5$, and there are no endothelial cells (tumor induced neovasculature). Yet, at later times, the densities of cancer cells are well fitted by Gaussian distributions.

Table 2

Parameters for the model with treatment.

\begin{tabular}{llll}
\hline Parameters & Values & Unity & Reference \\
\hline$D_{3}$ & $10^{-5}$ & $(\mathrm{~mm})^{2} \times$ day $^{-1}$ & {$[39]$} \\
$\lambda$ & 4.16 & day $^{-1}$ & {$[24]$} \\
$\mu$ & 8 & day $^{-1}$ & {$[27]$} \\
$\nu$ & $7 \times 10^{-2}$ & day $^{-1}$ & {$[24]$} \\
$\eta$ & 1 & day $^{-1}$ & $\eta \sim v$ \\
$a$ & $2 \times 10^{3}$ & mass $\times \mathrm{mm}^{-1}$ & {$[27]$} \\
$b$ & $5 \times 10^{5}$ & mass $\times \mathrm{mm}^{-1}$ & {$[27]$} \\
$c$ & $2 \times 10^{3}$ & mass $\times \mathrm{mm}^{-1}$ & {$[27]$} \\
$\tau$ & 0.125 & day & {$[24]$} \\
\hline
\end{tabular}

densities increase slowly, despite chemotherapy (MTD or metronomic), but only in the density-dependent drug delivery mode. Since endothelial cells support tumor growth, this is the primary reason that uniform drug delivery leads to increased therapeutic efficacy.

\section{Discussion}

Considering the complexity and nonlinearities involved in cancer progression and its interaction with therapeutics, mathematical models are valuable tools that provide quantitative understanding of the major mechanisms controlling tumor-drug dynamics and can aid in designing new protocols that enhance the success of anti-cancer therapies. Here, we 

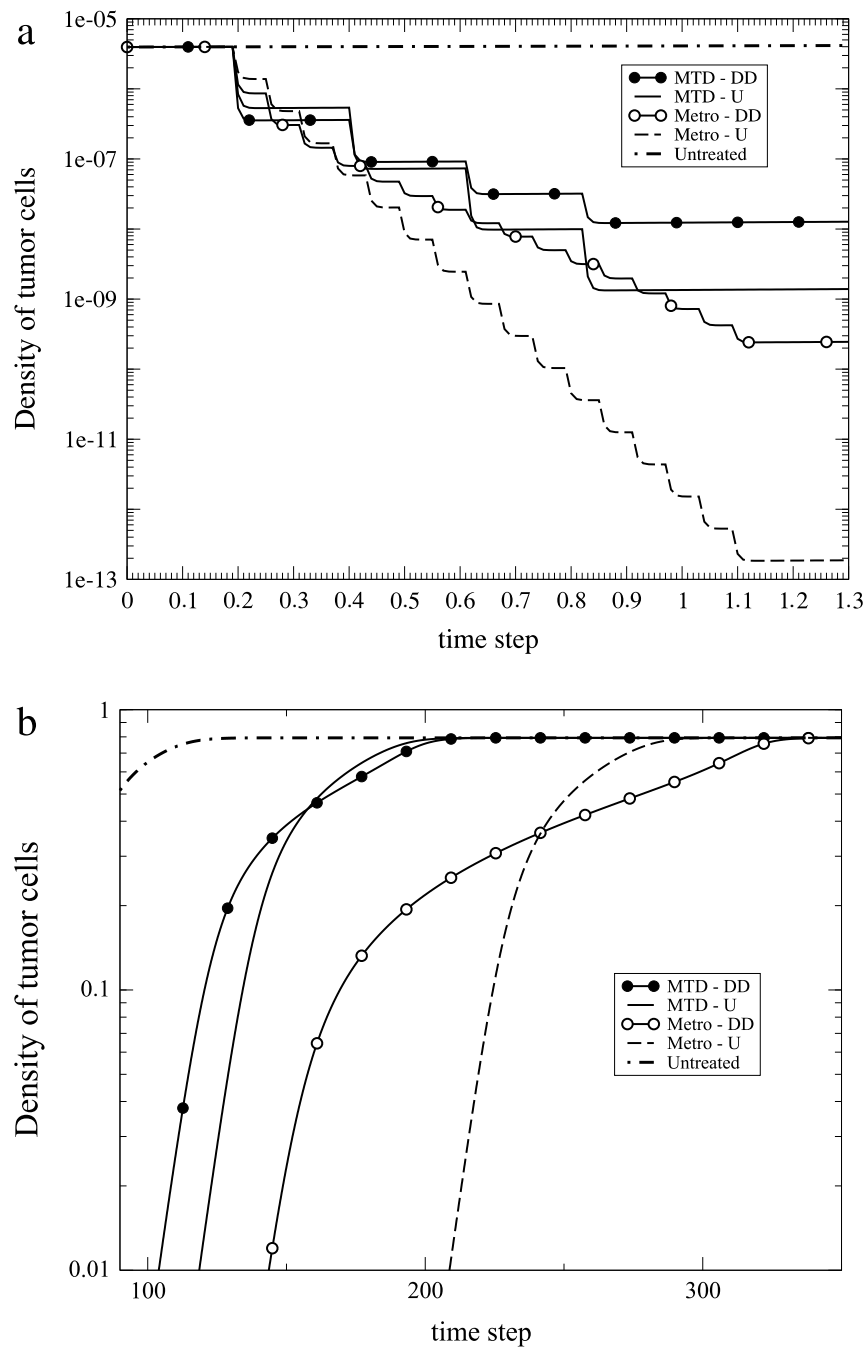

Fig. 4. (a) Evolution in time of densities of tumor cells on the interval $\bar{\Omega}=[0,1]$ during MTD and metronomic chemotherapies. (b) The same as in (a) but after the end of these treatments.

proposed and studied through analytical and numerical methods a population dynamics model in which normal and cancer cells compete for resources, the malignant cells induce the proliferation of endothelial cells and a chemotherapeutic drug with cytotoxic and antiangiogenic effects is used to treat a growing tumor. Our major aim was to evaluate the effects of metronomic and the MTD chemotherapy schedules on tumor treatment outcomes comparatively.

The major results of the present study are the following: the linear stability analysis demonstrates that the tumor population is eradicated without treatment only if normal cells are stronger competitors, i.e., $\alpha_{12}>k_{1} / k_{2}$, where $k_{1}$ and $k_{2}$ are the tissue's carrying supports for cancer and normal cells, respectively. Therefore, at least in their tissue of origin, cancer cells must acquire traits that increase the ratio $k_{1} / k_{2}$. Resistance to programmed cell death (apoptosis) or to hypoxia, via a shift to an anaerobic metabolism, are examples of such traits. Under therapy, this threshold value changes to

$$
\alpha_{12}^{*}=\frac{1-\frac{\mu}{r_{1}} \frac{q}{a \lambda+q}}{1-\frac{v}{r_{2}} \frac{q}{b \lambda+q}}\left(\frac{k_{1}}{k_{2}}\right),
$$

which depends on the functional responses of cancer and normal cells to the chemotherapeutic drug. Thus, in order to be efficacious, the drug must decrease $\alpha_{12}^{*}$ - a goal that can be achieved by increasing $\mu$ and decreasing $\nu$, i.e., by enhancing the drug's selectivity for cancer cells. Packaging clinically approved drugs into nanoscale delivery vehicles is a promising road toward enhancing selectivity for cancer cells. It is worth mentioning that our stability analysis reveals that cell replication rates affect the threshold $\alpha_{12}^{*}$. Nevertheless, the effect is weak for similar $r_{1}$ and $r_{2}$, as is normally the case. However, this result contrasts with the problems of two species competing for resources [40] and allelopathic suppression coupled to interspecific competition [41], in which the regimes of coexistence and extinction are determined only by the parameters controlling competition and functional responses to the allelochemicals. 

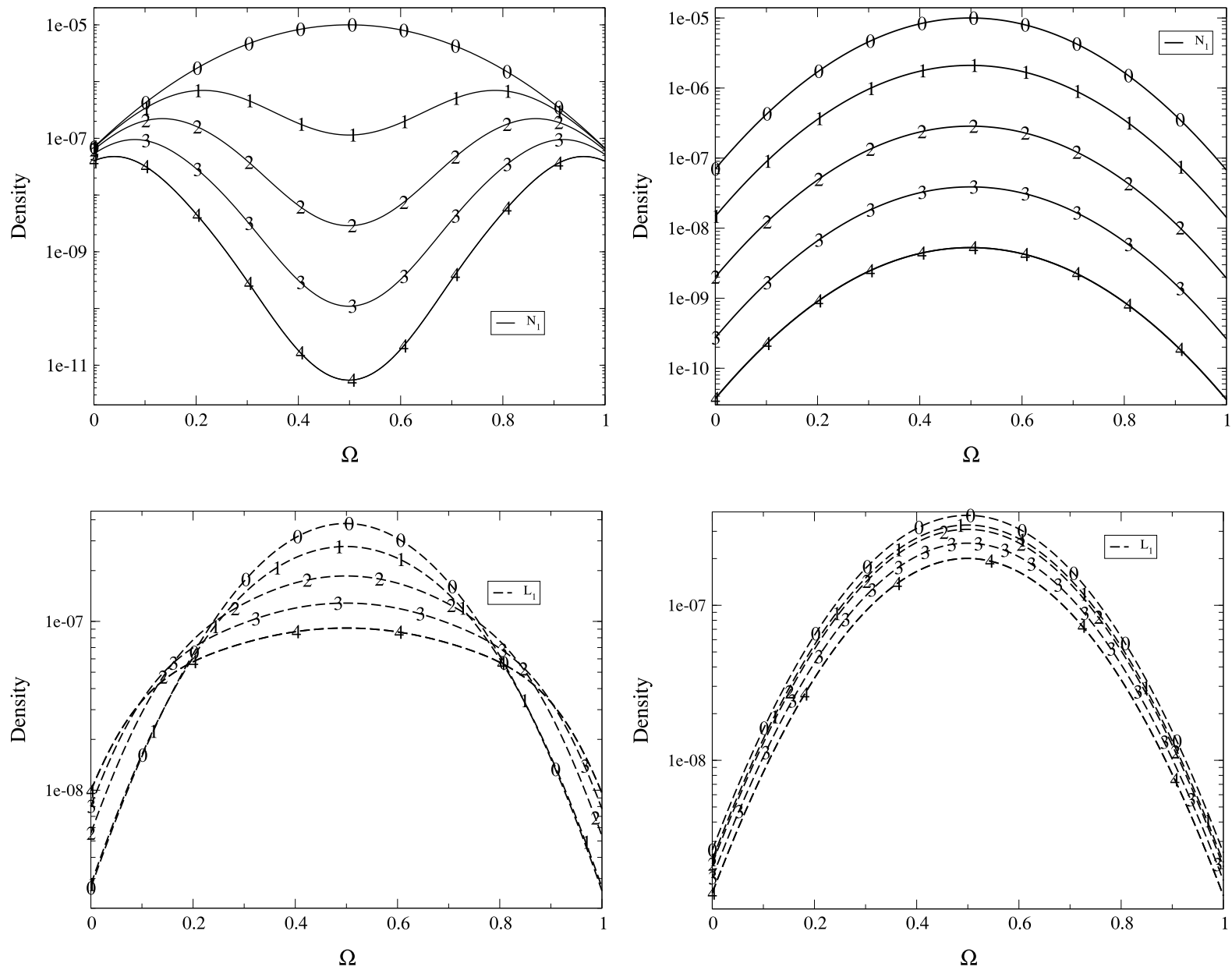

Fig. 5. Spatial density distributions for cancer $\left(N_{1}\right)$ and endothelial $\left(L_{1}\right)$ cells at distinct times during MTD chemotherapy under distinct drug delivery methods: density-dependent (on the left) and uniform (on the right). The different times are indicated above the curves.

Since continuous drug administration is clinically infeasible due to the highly deleterious side effects on normal cells, it is imperative to evaluate the effectiveness of metronomic and MTD chemotherapies comparatively. Our simulations indicate that the metronomic protocol is more efficacious in decreasing the density of tumor cells than the MTD protocol. Furthermore, metronomic chemotherapy combined with uniform drug delivery is the best strategy for reducing tumor density during treatment. However, in a metronomic protocol, it is density-dependent drug delivery, not the uniform mode, that most prolongs a patient's survival time post-therapy. These results engender a dilemma: uniform drug delivery improves treatment, but allows the possibility of a quick return for a non-eradicated tumor.

As shown in Fig. 4, the density of tumor cells can be dramatically reduced along chemotherapy, particularly under the metronomic protocol. However, from the mathematical viewpoint, the tumor was not eradicated and it regrows up to large densities finished the treatment. Biologically, such very low densities can effectively mean either tumor eradication or that only a ten of cancer cells survived. This is, in fact, a difficulty faced by continuous models in the study of eradication events in population dynamics.

A widely held viewpoint is that the treatment of cancer, owing to its metastatic spread throughout the organism, requires therapies based on medicines capable of efficient and uniform systemic delivery. One way to ensure uniform drug distribution throughout the tumor is to utilize angiogenic drugs that temporarily normalize the abnormal structure and function of the tumoral vasculature [42-44]. The rationale is appealing: repairing the vascular delivery system both increases and homogenizes the distribution of drug and oxygen throughout the tumor. Our simulational results (e.g., in Fig. 6) exemplify the convenience of combining drugs that normalize tumoral vasculature and cytotoxic therapies. However, the timing of drug combination is delicate: the normalization of the vasculature must precede the cytotoxic therapy in order to generate the most uniform initial distribution of endothelial cells. If this initial normalization is achieved, its benefit will last the duration of the treatment. This finding is in contrast with those obtained in Ref. [45]. Alarcon et al. indicate that a subtle interplay between vascular structural adaptation, oxygen supply and cancer cell dynamics promotes the emergence of a window of opportunity during which there occurs a minor improvement in tumoral response to cytotoxic drugs. The 

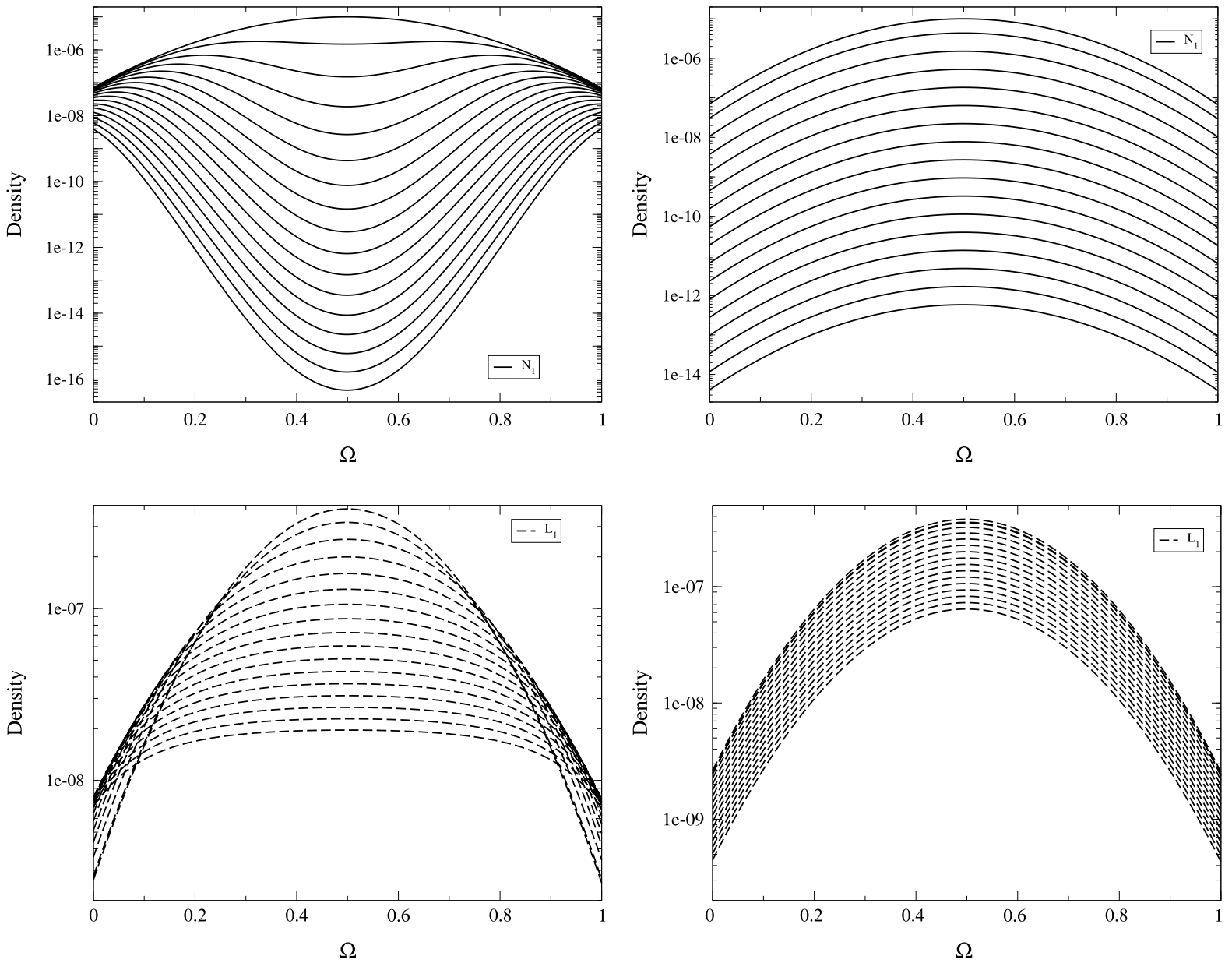

Fig. 6. The same information as in Fig. 5, but with regard to the metronomic protocol.

basic mechanism responsible for the better, but transient, therapeutic outcome is the increased fraction of proliferating cancer cells in tumors supported by the normalized vasculature. The anti-VEGFR drug leads to increased hypoxia, massive VEGF secretion and increased cell death inside the tumor. In turn, greater amounts of VEGF induce vessel remodeling to mitigate oxygen depletion within the hypoxic regions. This, in conjunction with the reduced oxygen demand elicited by cell death, produces the temporary expansion of the proliferative subpopulation susceptible to the drug. However, after this transient vascular normalization, other tumoral regions become hypoxic, and new adaptations of the vasculature close the window of opportunity.

Finally, as previously mentioned, our simulations also target a clinically relevant feature: tumor release after a failed chemotherapy depends on the drug delivery mode used over the course of the treatment. It is faster in a uniform than in a density-dependent mode. The reason is that uniform distribution of a cytotoxic drug produces a homogeneous level of tumoral and vascular inhibition. Once the therapy has ceased, there are few larger regions that are poorly vascularized, hypoxic and populated by quiescent cancer cells. The relative abundance of nutrients and reduced oxygen demand lead to a fast expansion of the proliferative subpopulation. In contrast, in density-dependent drug delivery, only the tissue close to the vasculature experiences homogeneous level of inhibition. Near the blood vessels, tumor and endothelial cells are practically eradicated, but larger regions that are poorly vascularized, and thereby hypoxic and populated by quiescent cancer cells, remain. Within these poorly vascularized regions, the tumor grows slowly.

\section{Conclusion}

The efficacies of metronomic and MTD anti-cancer chemotherapies were evaluated comparatively through numerical simulations of a PDE-based model. The model takes into account the competition between normal and tumor cells and the angiogenesis induced by malignant cells. The results indicate that the metronomic protocol is more effective in prolonging a patients life than the MTD protocol. Furthermore, uniform drug delivery combined with the metronomic protocol promotes the greatest tumor remission over the length of the treatment. However, if failed, metronomic therapy with uniform drug delivery leads to a faster regrowth of a non-eradicated tumor, thus engendering a clinically relevant dilemma. 


\section{Acknowledgments}

RTG was supported by CAPES; MLM was supported by FAPEMIG: APQ-04232-10 and CNPq: 306024/2013-6; PFAM was supported by \#2013/08133-0 São Paulo Research Foundation (FAPESP); \#2009/15098-0 São Paulo Research Foundation (FAPESP); FUNDUNESP: 1886/009-13.

\section{References}

[1] R. Siegel, E. Ward, O. Brawley, A. Jermal, Cancer statistics, 2011: the impact of eliminating socioeconomic and racial disparities on premature cancer deaths, CA Cancer J. Clin. 61 (4) (2011) 212-236.

[2] C.J. Murray, A.D. Lopez, Alternative projections of mortality and disability by cause, 1990-2020: global burden of disease study, Lancet 349 (9064) (1997) $1498-1504$.

3] WHO, Cancer, http://http://www.who.int/topics/cancer/en/(Sep. Acess: March, 2015).

[4] S. Baruchel, D. Stempak, Low-dose metronomic chemotherapy: myth or truth? Onkologie 29 (2006) 305-307.

[5] N. André, D. Barbolosi, F. Billy, G. Chapuisat, F. Hubert, E. Grenier, A. Rovini, Mathematical model of cancer growth controled by metronomic chemotherapies, ESAIM: Proceed 41 (2013) 77-94.

[6] K. Lien, S. Georgsdottir, L. Sivanathan, K. Chan, U. Emmenegger, Low-dose metronomic chemotherapy: a systematic literature analysis, Eur. J. Cancer 49 (16) (2013) 3387-3395.

[7] M.A.J. Chaplain, A. Matzavinos, Mathematical modelling of spatio-temporal phenomena in tumour immunology, in: Tutorials in Mathematical Biosciences III, vol. 1872, Springer, The Netherlands, 2006, pp. 131-183.

[8] H.M. Yang, Mathematical modeling of solid cancer growth with angiogenesis, Theor. Biol. Med. Model. 9 (2) (2012) 3-39.

[9] E.A. Reis, L.B.L. Santos, S.T.R. Pinho, A cellular automata model for avascular solid tumor growth under the effect of therapy, Physica A 388 (7) (2009) $1303-1314$

[10] S.C. Ferreira Jr., M.L. Martins, M.J. Vilela, A growth model for primary cancer, Physica A 261 (1998) 569-580.

[11] S.C. Ferreira Jr., M.L. Martins, M.J. Vilela, A growth model for primary cancer (ii). new rules, progress curves and morphology transitions, Physica A 272 (1999) 245-256.

[12] K.R. Fister, J.C. Panetta, Optimal control applied to competing chemotherapeutic cell-kill strategies, SIAM J. Appl. Math. 63 (6) (2003) 1954-1971.

[13] K. Madhavi, T. Rao, P.R.S. Reddy, Optimal drug administration for cancer chemotherapy through stochastic programming, AJAMMS 2 (1) (2013) 37-45

[14] U. Ledzewicz, H. Schattler, M.R. Gahrooi, S.M. Dehkordi, On the mtd paradigm and optimal control for multi drug cancer chemotherapy, Math. Biosci. Eng. 10 (3) (2013) 803-819.

[15] G.S. Stamatakos, E.A. Kolokotroni, D.D. Dionysiou, E.C. Georgiadi, C. Desmedt, An advanced discrete state-discrete event multiscale simulation model of the response of a solid tumor to chemotherapy: mimicking a clinical study, J. Theoret. Biol. 266 (1)(2010) 124-139.

[16] M.L. Martins, S.C. Ferreira Jr. M.J. Vilela, Multiscale models for biological systems, Curr. Opin. Colloid Interface 15 (2010) 18-23.

[17] P. Hahnfeldt, D. Panigrahy, J. Folkman, L. Hlatky, Tumor development under angiogenic signaling: a dynamical theory of tumor growth, treatment response, and postvascular dormancy, Cancer Res. 59 (19) (1999) 4770-4775.

[18] A. d'Onofrio, A. Gandolfi, Tumour eradication by antiangiogenic therapy: analysis and extensions of the model by Hahnfeldt et al. (1999), Math. Biosci. 191 (2) (2004) 159-184.

[19] S.T.R. Pinho, F.S. Bacelar, R.F.S. Andrade, H.I. Freedman, A mathematical model for the effect of anti-angiogenic therapy in the treatment of cance tumours by chemotherapy, Nonlinear Anal. RWA 14 (1) (2013) 815-828.

[20] C. Phipps, Combination of chemotherapy and antiangiogenic therapies: a mathematical modelling approach (Master's thesis), University of Waterloo, Waterloo, 2009

[21] D. Majumder, Tumor angiogenesis based analytical model for the assessment of MCT and MTD chemotherapeutic strategies in cancer, J. Biol. Syst. 18 (4) (2010) 749-761.

[22] S. Benzekry, N. André, A. Benabdallah, J. Ciccolini, C. Faivre, F. Hubert, D. Barbolosi, Modelling the impact of anticancer agents on metastatic spreading Math. Model. Nat. Phenom. 7 (1) (2012) 306-366.

[23] C. Faivre, D. Barbolosi, E. Pasquier, N. André, A mathematical model for the administration of temozolomide: comparative analysis of conventional and metronomic chemotherapy regimens, Cancer Chemother. Pharm. 71 (4) (2013) 1013-1019.

[24] D.S. Rodrigues, P.F.A. Mancera, S.T.R. Pinho, Accessing the antiangiogenic effect of metronomic chemotherapy through a simple mathematical model, 2014. Preprint.

[25] D. Hanahan, G. Bergers, E. Bergsland, Less is more, regularly: metronomic dosing of cytotoxic drugs can target tumor angiogenesis in mice, J. Clin. Invest. 105 (8) (2000) 1045-1047.

[26] R.S. Kerbel, B.A. Kamen, The anti-angiogenic basis of metronomic chemotherapy, Nat. Rev. Cancer 4 (2004) 423-436.

[27] D.S. Rodrigues, P.F.A. Mancera, Mathematical analysis and simulations involving chemotherapy and surgery on large human tumours under a suitable cell-kill functional response, Math. Biosci. Eng. 10 (1)(2013) 221-234.

[28] D. Ribatti, Cancer stem cells and tumor angiogenesis, Cancer Lett. 321 (2012) 13-17.

[29] R. Bjerkvig, M. Johansson, H. Miletic, S.P. Niclou, Cancer stem cells and angiogenesis, Sem. Cancer Biol. 19 (2009) 279-284.

[30] L. Ricci-Vitiani, R. Pallini, M. Biffoni, M. Todaro, G. Invernici, T. Cenci, G. Maira, E.A. Parati, G. Stassi, L.M. Larocca, R. De Maria, Tumour vascularization via endothelial differentiation of glioblastoma stem-like cells, Nature 468 (2010) 824-828; Nature 477 (2011) 238 (corrigendum).

[31] S. Bao, Q. Wu, S. Sathornsumetee, Y. Hao, Z. Li, A.B. Hjelmeland, Q. Shi, R.E. McLendon, D.D. Bigner, J.N. Rich, Stem cell-like glioma cells promote tumor angiogenesis through vascular endothelial growth factor, Cancer Res. 66 (2006) 7843-7848.

[32] A.R.A. Anderson, M.A.J. Chaplain, Continuous and discrete mathematical models of tumor-induced angiogenesis, Bull. Math. Biol. 60 (5) (1998) $857-899$.

[33] R. Martin, K.L. Teo, Optimal Control of Drug Administration in Cancer Chemotherapy, World Scientific, Singapore, 1993.

[34] P.K. Burgess, P.M. Kulesa, J.D. Murray, E.C. Alvord Jr., The interaction of growth rates and diffusion coefficients in a three-dimensional mathematical model of gliomas, J. Neuropathol. Exp. Neurol. 56 (6) (1997) 704-713.

[35] J.S. Spratt, J.S. Meyer, J.A. Spratt, Rates of growth of human neoplasms: part II, J. Surg. Oncol. 61 (1) (1996) 68-83.

[36] M.A.J. Chaplain, S.R. McDougall, A.R.A. Anderson, Mathematical modeling of tumor-induced angiogenesis, Annu. Rev. Biomed. Eng. 8 (2006) $233-257$.

37] R.A. Weinberg, The Biology of Cancer, Garland Science, New York, 2006.

[38] S. Friberg, S. Mattson, On the growth rates of human malignant tumors: implications for medical decision making, J. Surg. Oncol. 65 (1997) $284-297$.

[39] P.J. Robinson, S.I. Rapoport, Model for drug uptake by brain tumors: effects of osmotic treatment and of diffusion in brain, J. Cereb. Blood Flow Metab. $10(2)(1990)$ 153-161

[40] N.F. Britton, Essential Mathematical Biology, Springer-Verlag, London, 2005.

[41] A.C. Fassoni, M.L. Martins, Mathematical analysis of a model for plant invasion mediated by allelopathy, Ecol. Complexity 18 (2014) 49-58.

[42] R.K. Jain, Normalization of tumor vasculature: an emerging concept in antiangiogenic therapy, Science 307 (5706) (2005) 58-62.

[43] V.P. Chauhan, J.D. Martin, H. Liu, D.A. Lacorre, S.R. Jain, S.V. Kozin, T. Stylianopoulos, A.S. Mousa, X. Han, P. Adstamongkonkul, Z. Popović, P. Huang, M.G. Bawendi, Y. Boucher, R.K. Jain, Angiotensin inhibition enhances drug delivery and potentiates chemoterapy by decompressing tumor blood vessels, Nature Commun. 4 (2013) 2516.

[44] R.K. Jain, Normalizing tumor microenvironment to treat cancer: bench to bedside to biomarkers, J. Clin. Oncol. 31 (17) (2013) $2205-2218$

[45] T. Alarcón, M.R. Owen, H.M. Byrne, P.K. Maini, Multiscale modelling of tumour growth and therapy: the influence of vessel normalisation on chemotherapy, Comput. Math. Methods Med. 7 (2-3)(2006) 85-119. 